\title{
Center of Twisted Graded Hecke Algebras for Homocyclic Groups ${ }^{\star}$
}

\author{
Wee Liang $G A N^{\dagger}$ and Matthew HIGHFIELD ${ }^{\ddagger}$ \\ $\dagger$ University of California, Riverside, CA 92521, USA \\ E-mail:wlgan@math.ucr.edu \\ $\ddagger$ Pepperdine University, Malibu, CA 90263, USA \\ E-mail: matthew.highfield@pepperdine.edu
}

Received March 31, 2014, in final form October 10, 2014; Published online October 15, 2014

http://dx.doi.org/10.3842/SIGMA.2014.098

\begin{abstract}
We determine explicitly the center of the twisted graded Hecke algebras associated to homocyclic groups. Our results are a generalization of formulas by M. Douglas and B. Fiol in [J. High Energy Phys. 2005 (2005), no. 9, 053, 22 pages].
\end{abstract}

Key words: twisted graded Hecke algebra; homocyclic group

2010 Mathematics Subject Classification: 20C08

\section{Main results}

The notion of twisted graded Hecke algebras was introduced by S. Witherspoon in [10]; they are variants of the graded Hecke algebras of V. Drinfel'd [4] and G. Lusztig [6] (see also [7]) and twisted symplectic reflection algebras of T. Chmutova [2]. To a finite dimensional complex vector space $V$, a finite subgroup $G$ of $\mathrm{GL}(V)$, and a 2-cocycle $\alpha$ of $G$, the associated twisted graded Hecke algebra $\mathrm{H}$ is, by definition, a Poincaré-Birkhoff-Witt deformation of the crossedproduct algebra $S V \#_{\alpha} G$, where $S V$ denotes the symmetric algebra of $V$. The center of $S V \#_{\alpha} G$ is $(S V)^{G}$, and it is a natural question to determine the center of $\mathrm{H}$. In the non-twisted case, the center of the graded Hecke algebra associated to a finite real reflection group was determined by G. Lusztig in [5, Theorem 6.5]. In this paper, we determine the center of $\mathrm{H}$ for the twisted graded Hecke algebra in [10, Example 2.16], where $V=\mathbb{C}^{n}$ and $G$ is isomorphic to a homocyclic group $(\mathbb{Z} / \ell \mathbb{Z})^{n-1}$. (By a homocyclic group, we mean a direct product of cyclic groups of the same order.) In this example, the algebra $\mathrm{H}$ is finitely generated as a module over its center; the center of $\mathrm{H}$ therefore plays an important role in the representation theory of $\mathrm{H}$. We show that the center of $\mathrm{H}$ is generated by $n+1$ elements subject to one relation, which we determine explicitly. Our results are a generalization of formulas by M. Douglas and B. Fiol who considered the special case when $n=3$ in their paper $[3]$ on $\mathbb{C}^{3} /(\mathbb{Z} / \ell \mathbb{Z})^{2}$ orbifolds with discrete torsion.

We state our main results in this section and give the proofs in Section 2. We shall work over $\mathbb{C}$. Let $n$ be an integer $\geq 3$, and $\ell$ an integer $\geq 2$. Let $V=\mathbb{C}^{n}$ and let $x_{1}, \ldots, x_{n}$ be the standard basis of $V$. Let $G$ be the subgroup of $S L_{n}(\mathbb{C})$ consisting of all diagonal matrices $g$ satisfying $g^{\ell}=1$. Let $\zeta$ be a primitive $\ell$-th root of unity.

Notation 1.1. All subscripts are taken modulo $n$. For example, $x_{n+1}=x_{1}$.

\footnotetext{
${ }^{\star}$ This paper is a contribution to the Special Issue on New Directions in Lie Theory. The full collection is available at http://www.emis.de/journals/SIGMA/LieTheory2014.html
} 
For $i=1, \ldots, n$, let $g_{i}$ be the element of $G$ such that

$$
g_{i}\left(x_{j}\right)= \begin{cases}\zeta x_{j}, & \text { if } j=i, \\ \zeta^{-1} x_{j}, & \text { if } j=i+1, \\ x_{j}, & \text { else. }\end{cases}
$$

Observe that $g_{n}=g_{1}^{-1} \cdots g_{n-1}^{-1}$. We have an isomorphism $(\mathbb{Z} / \ell \mathbb{Z})^{n-1} \stackrel{\sim}{\longrightarrow} G$ defined by sending $(1,0, \ldots, 0), \ldots,(0, \ldots, 0,1)$ to $g_{1}, \ldots, g_{n-1}$, respectively.

Define the 2-cocycle $\alpha: G \times G \rightarrow \mathbb{C}^{\times}$of $G$ by

$$
\alpha\left(g_{1}^{i_{1}} \cdots g_{n-1}^{i_{n-1}}, g_{1}^{j_{1}} \cdots g_{n-1}^{j_{n-1}}\right)=\zeta^{-i_{1} j_{2}-i_{2} j_{3}-\cdots-i_{n-2} j_{n-1}} .
$$

If $E$ is an algebra, an action of $G$ on $E$ is a homomorphism $G \rightarrow \operatorname{Aut}(E)$. Recall that for any algebra $E$ and an action of $G$ on $E$, one has the crossed product algebra $E \#_{\alpha} G$. As a vector space, $E \#_{\alpha} G$ is $E \otimes \mathbb{C} G$; the product is defined by

$$
(r \otimes g)(s \otimes h)=\alpha(g, h) r(g \cdot s) \otimes g h
$$

for all $r, s \in E$ and $g, h \in G$. If $g, h \in G$, then we shall denote their product in $E \#_{\alpha} G$ by $g * h$; thus,

$$
g * h=\alpha(g, h) g h .
$$

One has, for any $i, j \in\{1, \ldots, n\}$ with $|i-j| \notin\{1, n-1\}$,

$$
g_{i+1} * g_{i}=\zeta g_{i} * g_{i+1}, \quad g_{i} * g_{j}=g_{j} * g_{i} .
$$

Let $t=\left(t_{1}, \ldots, t_{n}\right) \in \mathbb{C}^{n}$, and write $T V$ for the tensor algebra of $V$. Following [10, Example 2.16], we make the following definition.

Definition 1.2. Let $\mathrm{H}$ be the associative algebra defined as the quotient of $T V \#_{\alpha} G$ by the relations:

$$
x_{i} x_{i+1}-x_{i+1} x_{i}=t_{i} g_{i}, \quad x_{i} x_{j}-x_{j} x_{i}=0
$$

for all $i, j \in\{1, \ldots, n\}$ with $|i-j| \notin\{1, n-1\}$.

Remark 1.3. By [10, Theorem 2.10] and [10, Example 2.16], the algebra $\mathrm{H}$ in Definition 1.2 is a twisted graded Hecke algebra for $G$. (However, when $n>3$ and $\ell=2$, this is not the most general twisted graded Hecke algebra for $G$; see [10, Example 2.16] and [9, Example 5.1].)

Let $\mathbb{C}\left[y_{1}^{ \pm}, \ldots, y_{n}^{ \pm}\right]$be the algebra of Laurent polynomials in the variables $y_{1}, \ldots, y_{n}$. The group $G$ acts on $\mathbb{C}\left[y_{1}^{ \pm}, \ldots, y_{n}^{ \pm}\right]$by

$$
g_{i} y_{1}^{p_{1}} \cdots y_{n}^{p_{n}}=\zeta^{p_{i}-p_{i+1}} y_{1}^{p_{1}} \cdots y_{n}^{p_{n}}
$$

for all $i \in\{1, \ldots, n-1\}$ and $p_{1}, \ldots, p_{n} \in \mathbb{Z}$.

Proposition 1.4. There is an injective homomorphism

$$
\Theta: \mathrm{H} \longrightarrow \mathbb{C}\left[y_{1}^{ \pm}, \ldots, y_{n}^{ \pm}\right] \#_{\alpha} G
$$

such that

$$
\begin{aligned}
& \Theta\left(x_{i}\right)=y_{i}-\left(\frac{\zeta t_{i}}{\zeta-1}\right) y_{i+1}^{-1} g_{i}, \\
& \Theta\left(g_{i}\right)=g_{i}
\end{aligned}
$$

for all $i \in\{1, \ldots, n\}$. 
Let

$$
\begin{aligned}
& I=\left\{\left\{i_{1}<\cdots<i_{k}\right\} \mid k \geq 0 ; i_{1}, \ldots, i_{k} \in\{1, \ldots, n\}\right\}, \\
& J=\left\{\left\{i_{1}<\cdots<i_{k}\right\} \in I|| i_{r}-i_{s} \mid \notin\{1, n-1\} \text { for all } r, s\right\} .
\end{aligned}
$$

Define the elements $\delta, \varepsilon_{1}, \ldots, \varepsilon_{n}$ of $\mathbb{Z}^{n}$ by

$$
\delta=(1,1, \ldots, 1), \quad \varepsilon_{1}=(1,1,0, \ldots, 0), \quad \varepsilon_{2}=(0,1,1,0, \ldots), \quad \ldots, \quad \varepsilon_{n}=(1,0, \ldots, 0,1) .
$$

Notation 1.5. For any variables $\omega_{1}, \ldots, \omega_{n}$ and $p=\left(p_{1}, \ldots, p_{n}\right) \in \mathbb{Z}^{n}$, we denote by $\omega^{p}$ the expression $\omega_{1}^{p_{1}} \cdots \omega_{n}^{p_{n}}$.

We shall set

$$
\tau_{i}=\frac{t_{i}}{\zeta-1} \quad \text { for } \quad i=1, \ldots, n-1, \quad \tau_{n}=\frac{\zeta t_{n}}{\zeta-1} .
$$

Define the element $w \in \mathrm{H}$ by

$$
w=\sum_{\left\{i_{1}<\cdots<i_{k}\right\} \in J} \tau_{i_{1}} \cdots \tau_{i_{k}} x^{\delta-\varepsilon_{i_{1}}-\cdots-\varepsilon_{i_{k}}} g_{i_{1}} * \cdots * g_{i_{k}} .
$$

Example 1.6. If $n=3$, then

$$
w=x_{1} x_{2} x_{3}+\tau_{1} x_{3} g_{1}+\tau_{2} x_{1} g_{2}+\tau_{3} x_{2} g_{3}=x_{1} x_{2} x_{3}+\frac{1}{\zeta-1}\left(t_{1} x_{3} g_{1}+t_{2} x_{1} g_{2}+\zeta t_{3} x_{2} g_{3}\right) .
$$

In particular, if $n=3$ and $\ell=2$, the formula for $w$ is in [1, Lemma 7.1].

Theorem 1.7. The center of $\mathrm{H}$ is generated as an algebra by $x_{1}^{\ell}, \ldots, x_{n}^{\ell}$, and $w$.

Let $\mathbf{Z}$ be the center of $\mathrm{H}$. For $r=0, \ldots,\lfloor\ell / 2\rfloor$, set

$$
\nu_{r}=(-1)^{r} \frac{\ell}{\ell-r}\left(\begin{array}{c}
\ell-r \\
r
\end{array}\right)
$$

and set

$$
\widetilde{\tau}_{i}=\tau_{i}^{\ell} \quad \text { for } \quad i=1, \ldots, n-1, \quad \widetilde{\tau}_{n}=(-1)^{n(\ell-1)} \tau_{n}^{\ell} .
$$

We define a polynomial $F$ in the $n+1$ variables $a_{1}, \ldots, a_{n}$ and $b$ by

$$
F=\sum_{\left\{i_{1}<\cdots<i_{k}\right\} \in J} \widetilde{\tau}_{i_{1}} \cdots \widetilde{\tau}_{i_{k}} a^{\delta-\varepsilon_{i_{1}}-\cdots-\varepsilon_{i_{k}}}-\sum_{r=0}^{\lfloor\ell / 2\rfloor}(-1)^{n r} \zeta^{(n-2) r} \nu_{r}\left(\tau_{1} \cdots \tau_{n}\right)^{r} b^{\ell-2 r} .
$$

Corollary 1.8. The assignment

$$
a_{i} \mapsto x_{i}^{\ell} \quad \text { for } \quad i=1, \ldots, n, \quad b \mapsto w
$$

defines an isomorphism

$$
\mathbb{C}\left[a_{1}, \ldots, a_{n}, b\right] /(F) \stackrel{\sim}{\longrightarrow} \mathrm{Z} .
$$

In the undeformed case, when $t_{1}=\cdots=t_{n}=0$, the polynomial $F$ is equal to $a_{1} \cdots a_{n}-b^{\ell}$. 


\section{Proof of main results}

Proof of Proposition 1.4. For $i=1, \ldots, n-1$, we define $\Theta\left(x_{i}\right), \Theta\left(x_{n}\right)$, and $\Theta\left(g_{i}\right)$ by (1.1) and (1.2). It follows from a straightforward verification that $\Theta$ is a well-defined homomorphism.

It remains to see that $\Theta$ is injective. Observe that $\mathrm{H}$ is spanned by the monomials $x^{p} g$ for $p=\left(p_{1}, \ldots, p_{n}\right) \in \mathbb{Z}^{n}$ and $g \in G$, where $p_{1}, \ldots, p_{n} \geq 0$. We call $p_{1}+\cdots+p_{n}$ the total degree of the monomial $x^{p} g$. The image of $x^{p} g$ under $\Theta$ is the sum of $y^{p} g$ with terms of strictly smaller total degrees. Therefore, if $\alpha \in \mathrm{H}$ is nonzero, we can write it as a sum $\alpha_{0}+\alpha_{1}+\cdots$, where $\alpha_{k}$ is a linear combination of monomials $x^{p} g$ with total degree $k$. If $k$ is the maximal integer with $\alpha_{k}$ nonzero, then $\Theta\left(\alpha_{k}\right)$ is nonzero, and hence $\Theta(\alpha)$ is also nonzero.

Remark 2.1. It follows from Proposition 1.4 that the monomials $x_{1}^{p_{1}} \cdots x_{n}^{p_{n}} g$ for non-negative integers $p_{1}, \ldots, p_{n}$ and $g \in G$ form a basis for $\mathrm{H}$ (called the $\mathrm{PBW}$ basis of $\mathrm{H}$ ). This was first proved in [10, Example 2.16] using [10, Theorem 2.10].

We have an increasing filtration on $\mathrm{H}$ defined by setting $\operatorname{deg}\left(x_{i}\right)=1$ and $\operatorname{deg}(g)=0$ for all $i \in\{1, \ldots, n\}, g \in G$. It is immediate from Remark 2.1 that the natural homomorphism $S V \#_{\alpha} G \rightarrow \mathrm{grH}$ is an isomorphism, where grH denotes the associated graded algebra of $\mathrm{H}$.

The proof of (2.3) in the following lemma is the key calculation in this paper.

\section{Lemma 2.2 .}

(i) One has:

$$
\begin{aligned}
& \Theta\left(x_{i}^{\ell}\right)=y_{i}^{\ell}-\tau_{i}^{\ell} y_{i+1}^{-\ell}, \\
& \Theta\left(x_{n}^{\ell}\right)=y_{n}^{\ell}-(-1)^{n(\ell-1)} \tau_{n}^{\ell} y_{1}^{-\ell},
\end{aligned}
$$

for all $i \in\{1, \ldots, n-1\}$.

(ii) One has:

$$
\Theta(w)=y_{1} \cdots y_{n}+(-1)^{n} \zeta^{n-2} \tau_{1} \cdots \tau_{n} y_{1}^{-1} \cdots y_{n}^{-1}
$$

Proof. (i) To prove (2.1), we need to show that

$$
\underbrace{\left(y_{i}-\zeta \tau_{i} y_{i+1}^{-1} g_{i}\right) \cdots\left(y_{i}-\zeta \tau_{i} y_{i+1}^{-1} g_{i}\right)}_{\ell}=y_{i}^{\ell}-\tau_{i}^{\ell} y_{i+1}^{-\ell} .
$$

Since $g_{i} y_{i}=\zeta y_{i} g_{i}$ and $g_{i} y_{i+1}^{-1}=\zeta y_{i+1}^{-1} g_{i}$, the product on the left hand side of (2.4) is a linear combination of $y_{i}^{k} y_{i+1}^{k-\ell} g_{i}^{\ell-k}$ for $k=0,1, \ldots, \ell$. Moreover, the coefficient of $y_{i}^{k} y_{i+1}^{k-\ell} g_{i}^{\ell-k}$ in this linear combination is the same as the coefficient of $u^{k}$ when we expand the product

$$
\left(u-\zeta^{\ell} \tau_{i}\right)\left(u-\zeta^{\ell-1} \tau_{i}\right) \cdots\left(u-\zeta \tau_{i}\right)
$$

in the polynomial ring $\mathbb{C}[u]$. Since the polynomial in $(2.5)$ is equal to $u^{\ell}-\tau_{i}^{\ell}$, the identity $(2.1)$ follows. The proof of (2.2) is similar except that

$$
\underbrace{g_{n} * \cdots * g_{n}}_{\ell}=(-1)^{n(\ell-1)} \text {. }
$$

(ii) For any $h_{*}=\left\{h_{1}<\cdots<h_{j}\right\} \in I$, we let

$$
\begin{aligned}
& h_{*}^{\prime}=\left\{h_{r} \in h_{*} \mid h_{s}-h_{r} \in\{1,1-n\} \text { for some } s\right\}, \\
& \chi\left(h_{*}\right)=\left|\left\{h_{r} \in h_{*}^{\prime} \mid h_{r} \neq n\right\}\right|-\left|\left\{h_{r} \in h_{*}^{\prime} \mid h_{r}=n\right\}\right|,
\end{aligned}
$$




$$
E\left(h_{*}\right)=\zeta^{\chi\left(h_{*}\right)} \tau_{h_{1}} \cdots \tau_{h_{j}} y^{\delta-\varepsilon_{h_{1}}-\cdots-\varepsilon_{h_{j}}} g_{h_{1}} * \cdots * g_{h_{j}} .
$$

Now suppose $i_{*}=\left\{i_{1}<\cdots<i_{k}\right\} \in J$. Let $D$ be the subset of $\{1, \ldots, n\}$ consisting of all $d$ such that $d \not \equiv i_{r}, i_{r}+1(\bmod n)$ for all $r$. We denote by $d_{1}<\cdots<d_{p}$ the elements of $D$. Then

$$
\begin{aligned}
& \Theta\left(\tau_{i_{1}} \cdots \tau_{i_{k}} x^{\delta-\varepsilon_{i_{1}}-\cdots-\varepsilon_{i_{k}}} g_{i_{1}} * \cdots * g_{i_{k}}\right) \\
& \quad=\tau_{i_{1}} \cdots \tau_{i_{k}}\left(y_{d_{1}}-\frac{\zeta t_{d_{1}}}{\zeta-1} y_{d_{1}+1}^{-1} g_{d_{1}}\right) \cdots\left(y_{d_{p}}-\frac{\zeta t_{d_{p}}}{\zeta-1} y_{d_{p}+1}^{-1} g_{d_{p}}\right) g_{i_{1}} * \cdots * g_{i_{k}} \\
& \quad=\tau_{i_{1}} \cdots \tau_{i_{k}} \sum_{S \subset D} Y_{d_{1}}(S) \cdots Y_{d_{p}}(S) g_{i_{1}} * \cdots * g_{i_{k}}
\end{aligned}
$$

where, for $r=1, \ldots, p$,

$$
Y_{d_{r}}(S)=\left\{\begin{array}{lll}
y_{d_{r}}, & \text { if } & d_{r} \notin S \\
-\zeta(\zeta-1)^{-1} t_{d_{r}} y_{d_{r}+1}^{-1} g_{d_{r}}, & \text { if } & d_{r} \in S .
\end{array}\right.
$$

Setting $h_{*}=i_{*} \cup S$, we obtain ${ }^{1}$

$$
\Theta\left(\tau_{i_{1}} \cdots \tau_{i_{k}} x^{\delta-\varepsilon_{i_{1}}-\cdots-\varepsilon_{i_{k}}} g_{i_{1}} * \cdots * g_{i_{k}}\right)=\sum_{\left\{h_{*} \in I \mid i_{*} \subset h_{*}-h_{*}^{\prime}\right\}}(-1)^{\left|h_{*}\right|-\left|i_{*}\right|} E\left(h_{*}\right) .
$$

Hence,

$$
\begin{aligned}
\Theta(w) & =\sum_{\left\{i_{1}<\cdots<i_{k}\right\} \in J} \Theta\left(\tau_{i_{1}} \cdots \tau_{i_{k}} x^{\delta-\varepsilon_{i_{1}}-\cdots-\varepsilon_{i_{k}}} g_{i_{1}} * \cdots * g_{i_{k}}\right) \\
& =\sum_{i_{*} \in J}\left(\sum_{\left\{h_{*} \in I \mid i_{*} \subset h_{*}-h_{*}^{\prime}\right\}}(-1)^{\left|h_{*}\right|-\left|i_{*}\right|} E\left(h_{*}\right)\right)=\sum_{h_{*} \in I}\left(E\left(h_{*}\right) \sum_{i_{*} \subset h_{*}-h_{*}^{\prime}}(-1)^{\left|h_{*}\right|-\left|i_{*}\right|}\right) .
\end{aligned}
$$

If $\left|h_{*}\right|=n$, then $h_{*}^{\prime}=h_{*}$. If $\left|h_{*}\right| \notin\{0, n\}$, then $h_{*}^{\prime} \neq h_{*}$. Therefore,

$$
E\left(h_{*}\right) \sum_{i_{*} \subset h_{*}-h_{*}^{\prime}}(-1)^{\left|h_{*}\right|-\left|i_{*}\right|}= \begin{cases}y_{1} \cdots y_{n} & \text { if }\left|h_{*}\right|=0, \\ (-1)^{n} \zeta^{n-2} \tau_{1} \cdots \tau_{n} y_{1}^{-1} \cdots y_{n}^{-1} & \text { if }\left|h_{*}\right|=n, \\ 0 & \text { else. }\end{cases}
$$

Proof of Theorem 1.7. It is easy to see that the center of $S V \#_{\alpha} G$ is the algebra of $G$-invariant elements $(S V)^{G}$ of $S V$, and moreover, the algebra $(S V)^{G}$ is generated by $x_{i}^{\ell}(i=1, \ldots, n)$ and $x_{1} \cdots x_{n}$.

Using Lemma 2.2, we see that

$$
\Theta\left(x_{i}^{\ell}\right) \quad \text { for } \quad i=1, \ldots, n, \quad \text { and } \quad \Theta(w)
$$

are in the center of $\mathbb{C}\left[y_{1}^{ \pm}, \ldots, y_{n}^{ \pm}\right] \#_{\alpha} G$. Since the homomorphism $\Theta$ is injective, the elements $x_{i}^{\ell}$ $(i=1, \ldots, n)$ and $w$ are in the center of $\mathbf{H}$. Since the principal symbols of $x_{1}^{\ell}, \ldots, x_{n}^{\ell}$ and $w$ in $S V \#_{\alpha} G$ are, respectively, $x_{1}^{\ell}, \ldots, x_{n}^{\ell}$ and $x_{1} \cdots x_{n}$, the theorem follows from a standard argument.

\footnotetext{
${ }^{1}$ Note that if $d_{r} \in S$ but $d_{r}+1 \in D-S$, then the term $g_{d_{r}}$ in $Y_{d_{r}}(S)$ appears on the left of the term $y_{d_{r}+1}$ of $Y_{d_{r}+1}(S)$ and one has $g_{d_{r}} y_{d_{r}+1}=\zeta^{-1} y_{d_{r}+1} g_{d_{r}}$. However, if $n \in S$ but $1 \in D-S$, then the term $g_{n}$ in $Y_{n}(S)$ already appears to the right of the term $y_{1}$ of $Y_{1}(S)$. This is the reason why the definition of $\tau_{n}$ differs from the corresponding definitions of $\tau_{1}, \ldots, \tau_{n-1}$ by a factor of $\zeta$.
} 
Proof of Corollary 1.8. Let $\widetilde{a}_{1}=\Theta\left(x_{1}^{\ell}\right), \ldots, \widetilde{a}_{n}=\Theta\left(x_{n}^{\ell}\right)$, and $\widetilde{b}=\Theta(w)$. By Lemma 2.2,

$$
\begin{aligned}
& \widetilde{a}_{i}=y_{i}^{\ell}-\widetilde{\tau}_{i} y_{i+1}^{-\ell} \quad \text { for } \quad i=1, \ldots, n, \\
& \widetilde{b}=y_{1} \cdots y_{n}+(-1)^{n} \zeta^{n-2} \tau_{1} \cdots \tau_{n} y_{1}^{-1} \cdots y_{n}^{-1} .
\end{aligned}
$$

By a calculation completely similar to the proof of (2.3), one has

$$
\sum_{\left\{i_{1}<\cdots<i_{k}\right\} \in J} \widetilde{\tau}_{i_{1}} \cdots \widetilde{\tau}_{i_{k}} \widetilde{a}^{\delta-\varepsilon_{i_{1}}-\cdots-\varepsilon_{i_{k}}}=\left(y_{1} \cdots y_{n}\right)^{\ell}+(-1)^{n \ell}\left(\tau_{1} \cdots \tau_{n}\right)^{\ell}\left(y_{1} \cdots y_{n}\right)^{-\ell} .
$$

We claim that we also have

$$
\sum_{r=0}^{\lfloor\ell / 2\rfloor}(-1)^{n r} \zeta^{(n-2) r} \nu_{r}\left(\tau_{1} \cdots \tau_{n}\right)^{r} \widetilde{b}^{\ell-2 r}=\left(y_{1} \cdots y_{n}\right)^{\ell}+(-1)^{n \ell}\left(\tau_{1} \cdots \tau_{n}\right)^{\ell}\left(y_{1} \cdots y_{n}\right)^{-\ell} .
$$

To see this, recall that the Chebyshev polynomials of the first kind are defined recursively by $T_{0}(\xi)=1, T_{1}(\xi)=\xi$, and

$$
T_{m}(\xi)=2 \xi T_{m-1}(\xi)-T_{m-2}(\xi) \text { for } m=2,3, \ldots
$$

It is well known (and can be easily proved by induction) that

$$
\begin{aligned}
& 2 T_{\ell}\left(\frac{\xi}{2}\right)=\sum_{r=0}^{\lfloor\ell / 2\rfloor} \nu_{r} \xi^{\ell-2 r}, \\
& 2 T_{\ell}\left(\frac{\xi+\xi^{-1}}{2}\right)=\xi^{\ell}+\xi^{-\ell} .
\end{aligned}
$$

By (2.8) and (2.9), one has the identity

$$
\xi^{\ell}+\xi^{-\ell}=\sum_{r=0}^{\lfloor\ell / 2\rfloor} \nu_{r}\left(\xi+\xi^{-1}\right)^{\ell-2 r}
$$

and hence the identity

$$
\xi^{\ell}+\varrho^{2 \ell} \xi^{-\ell}=\sum_{r=0}^{\lfloor\ell / 2\rfloor} \nu_{r} \varrho^{2 r}\left(\xi+\varrho^{2} \xi^{-1}\right)^{\ell-2 r}
$$

where $\xi$ and $\varrho$ are formal variables. By setting $\xi=y_{1} \cdots y_{n}$ and choosing $\varrho$ to be a square-root of $(-1)^{n} \zeta^{n-2} \tau_{1} \cdots \tau_{n}$, we obtain $(2.7)$.

By Proposition 1.4, Theorem 1.7, and the equations (2.6) and (2.7), the assignment (1.4) defines a surjective homomorphism

$$
\Phi: \mathbb{C}\left[a_{1}, \ldots, a_{n}, b\right] \rightarrow \mathrm{Z}
$$

such that $\Phi(F)=0$. Suppose $D \in \mathbb{C}\left[a_{1}, \ldots, a_{n}, b\right]$ and $\Phi(D)=0$. We can write

$$
D=\sum_{r=0}^{\ell-1} D_{r}\left(a_{1}, \ldots, a_{n}\right) b^{r}+R
$$

where $D_{r}\left(a_{1}, \ldots, a_{n}\right) \in \mathbb{C}\left[a_{1}, \ldots, a_{n}\right]$ for $r=0, \ldots, \ell-1$, and $R \in(F)$. Thus,

$$
\sum_{r=0}^{\ell-1} D_{r}\left(x_{1}^{\ell}, \ldots, x_{n}^{\ell}\right) w^{r}=0 \text {. }
$$


We claim that $D_{r}\left(a_{1}, \ldots, a_{n}\right)=0$ for all $r$. Suppose not; then let $m$ be the maximal integer such that $D_{m}\left(a_{1}, \ldots, a_{n}\right) \neq 0$. Let $x_{1}^{\ell p_{1}} \cdots x_{n}^{\ell p_{n}}$ be a monomial in $D_{m}\left(x_{1}^{\ell}, \ldots, x_{n}^{\ell}\right)$ with nonzero coefficient. Since $0 \leq m<\ell$, when we write the left hand side of (2.10) in terms of the PBW basis, the coefficient of $x_{1}^{\ell p_{1}+m} \cdots x_{n}^{\ell p_{n}+m}$ is nonzero, a contradiction. Hence, the kernel of $\Phi$ is $(F)$. This proves $(1.5)$.

Remark 2.3. When $n=3$, the algebra $\mathrm{H}$ is Morita equivalent to a deformed Sklyanin algebra $S_{\text {def }}$ defined by C. Walton in [8, Definition IV.2]. More precisely, if $n=3$ and

$$
e=\frac{1}{\ell} \sum_{r=0}^{\ell-1} g_{1}^{r},
$$

one has $\mathrm{HeH}=\mathrm{H}$ and $e \mathrm{H} e \cong S_{\text {def }}$ where the parameters for $S_{\text {def }}$ (following the notations in [8, Definition IV.2]) are $a=1, b=\zeta, c=d_{i}=0$, and $e_{i}=-\zeta t_{i}$ for $i=1,2,3$. This follows from the observation that, for $n=3$, setting $\phi_{i}=x_{i} g_{i+1}$, one has $\phi_{i} \phi_{i+1}-\zeta \phi_{i+1} \phi_{i}=\zeta t_{i}$ for all $i$. The algebra $S_{\text {def }}$ (with above parameters) was first studied by M. Douglas and B. Fiol, see $[3,(3.10)]$. Our formulas (1.1)-(1.2) are a generalization of $[3,(4.6)]$, and our equation (1.3) is a generalization of $[3,(4.7)]$. The formulas in (2.1)-(2.3) are generalizations of $[3,(4.8)]$.

\section{Acknowledgements}

We thank the referees for their many helpful comments.

\section{References}

[1] Căldăraru A., Giaquinto A., Witherspoon S., Algebraic deformations arising from orbifolds with discrete torsion, J. Pure Appl. Algebra 187 (2004), 51-70, math.KT/0210027.

[2] Chmutova T., Twisted symplectic reflection algebras, math.RT/0505653.

[3] Douglas M.R., Fiol B., D-branes and discrete torsion. II, J. High Energy Phys. 2005 (2005), no. 9, 053, 22 pages, hep-th/9903031.

[4] Drinfel'd V.G., Degenerate affine Hecke algebras and Yangians, Funct. Anal. Appl. 20 (1986), 58-60.

[5] Lusztig G., Cuspidal local systems and graded Hecke algebras. I, Inst. Hautes Études Sci. Publ. Math. (1988), 145-202.

[6] Lusztig G., Affine Hecke algebras and their graded version, J. Amer. Math. Soc. 2 (1989), 599-635.

[7] Ram A., Shepler A.V., Classification of graded Hecke algebras for complex reflection groups, Comment. Math. Helv. 78 (2003), 308-334, math.GR/0209135.

[8] Walton C.M., On degenerations and deformations of Sklyanin algebras, Ph.D. Thesis, University of Michigan, 2011.

[9] Witherspoon S., Skew derivations and deformations of a family of group crossed products, Comm. Algebra 34 (2006), 4187-4206, math.RA/0506154.

[10] Witherspoon S., Twisted graded Hecke algebras, J. Algebra 317 (2007), 30-42, math.RT/0506152. 